\title{
High Speed Wireless Internet Access: Combination of MPLS and BRAN HIPERLAN/2 Technologies
}

\author{
Kallikratidas Nitsos ${ }^{1}$, Tasos Dagiuklas ${ }^{1}$, and Hamed Al-Raweshidy ${ }^{2}$ \\ 1 INTRACOM S.A., Markopoulo Avenue, Peania 190 02, Attika, GREECE \\ Tel: (30-1) 6690368, Fax: (30-1) 6860312 \\ \{knit, ntan\}@intracom.gr \\ 2 University of Kent at Canterbury, Canterbury, Kent CT2 7NT, UK \\ Tel: (44-1227) 823396, Fax: (44-1227) 456084 \\ H.Al-Raweshidy@ukc.ac.uk
}

\begin{abstract}
This paper discusses the provision of High Speed Wireless Internet Access. It combines the MPLS technology for the core network and the next generation wireless BRAN HIPERLAN2 technology for the access part. The paper focuses on the interworking of MPLS within the HIPERLAN/2, through the design of a Convergence Layer. Two cases have been examined. In the first one, the AP is MPLS capable running a "light" version of the LDP protocol whereas in the second one MPLS is terminated at the edge router. The advantages and disadvantages of both approaches have been presented. Furthermore, the employment of MPLS to provide mobility management functionalities has been addressed. Two mobility management schemes have been examined. In the first one, MPLS is combined with Mobile IP to alleviate the problems of IP-in-IP encapsulation and triangular routing. In the second one, MPLS extensions have been proposed to provide mobility management services in an MPLS Administrative Domain.
\end{abstract}

\section{Introduction}

Multimedia services require strict QoS requirements in terms of loss and delay. The transmission of these services over IP-based networks, necessitates the forwarding of IP datagrams towards the destination host in a fast and efficient way. The above requirement has led the research community to adopt the Layer 3 switching (IP switching) concept. Several companies (e.g., Nokia IP [1], Cisco [2], Toshiba [3]) have proposed a proprietary IP switching scheme to provide faster routing of the IP datagrams. The IETF established in early 1997 the MPLS Working Group to unify and standardise the various IP switching proposals. The major goal of MPLS is the combination of the benefits of switching and IP routing [4]. On the other hand, the research community has recently paid attention towards the integration of Internet technology and wireless access systems, for the provision of Internet services over wireless/mobile devices. The objective of this paper is the provision of High Speed Broadband Wireless Internet Access by combining the benefits of next generation wireless access networks and MPLS. The driving forces have been the ETSI through the standardisation process for Broadband Radio Access Networks (BRAN- HIgh 
PErformance Radio LAN with broadband capabilities - HL/2) and IETF. The innovative aspects of the paper are: the interworking of MPLS within the BRAN HL/2, through the design of a Convergence Layer (CL) and extensions of MPLS to provide Mobility Management by i) combining Mobile IP and MPLS and ii) using the functionality of MPLS.

\section{MultiProtocol Label Switching (MPLS)}

MPLS integrates the label swapping forwarding paradigm with network layer routing. Thus it improves the price/performance of network layer routing, enhances the scalability of the network layer and increases the speed in the Internet [5], [6]. Within the MPLS Administrative Domain, a Label Edge Router (LER) is an MPLS node connecting an MPLS domain with a node outside of the domain. LER is the router that assigns labels and determines explicit routing paths. A Label Switch Router (LSR) is a node, which runs MPLS and is capable of forwarding packets based on labels. Label Distribution Protocol (LDP) is the main signaling protocol used in MPLS for assigning, managing, maintaining and distributing labels.

\section{BRAN / HIPERLAN 2}

ETSI has defined three types of broadband radio networks: HL/2 (25 Mbps typical data rate), HIPERACCESS (25 Mbps typical data rate) and HIPERLINK (up to 155 Mbps data rate). In this paper, we have concentrated in HL/2 systems [7]-[9], because the proposed system architecture includes mobility support functionality. HL/2 provides wireless access to different infrastructure networks (e.g. IP, ATM) by both moving and stationary terminals interacting with access points which, in turn, usually are connected to an IP, ATM or other type of backbone network. For the Data Link Control (DLC) addressing, the following of IDs have been used: a MAC-ID which identifies the terminal; a DLC Connection-ID (DLCC-ID) which identifies the connection within the terminal; an Access Point-ID (AP-ID) which identifies the AP Controller (APC) and AP Transceiver (APT), entities of the AP and a NET-ID which is the same for all APs belonging to the same core network.

\section{Interworking of BRAN/HIPERLAN2 with MPLS}

The interworking between the HL/2 DLC layer and the different network layers defined by the correspondent fora, requires the introduction of a CL between them [10]-[13]. Within the context of this paper, ATM is employed as a transport technology without using the ATM signalling and traffic management functionalities. Thus, the CL must convert the DLC-PDUs into ATM cells, and insert labels in them.

Two different approaches have been followed, i) AP and Edge Router (ER) are MPLS capable, ii) AP is MPLS non-capable and ER is MPLS capable. 
The term "MPLS capable" as applied to the AP means that through the employment of labels, LDP does not establish paths but inserts labels in order to pass BRAN-related information to the ER. Therefore, a "light version" of LDP runs on the AP. The operation of LDP on the ER remains the same, since Fixed Terminals (FTs) may be connected to the ER.

\subsection{Both AP and ER Are MPLS Capable}

Upstream means that data are transmitted from the MT towards the core network, while downstream is the opposite direction.

Upstream. The IP layer in the MT is integrated over the BRAN DLC layer through the CL. Peer-to-peer communication, between the AP and the MT takes place and in turn the AP communicates with the ER. Hence, the ER knows the IP addresses of the MTs (stores them in a table) attached (via the AP) to it.

The MT receives from the AP Information Elements (IE), containing the MAC-ID and the DLCC-ID that the AP assigns to the MT. The MT sends a DLC-PDU to the AP as defined by BRAN. On receipt of the DLC-PDU, the AP strips the FU, the SN and the CRC bits, to construct the DLC-SDU. It will read the CL-Tag field of the received DLC-PDU and will understand to which CL the frame must be sent. The rest of the process is shown in Fig.1-a. The CPCS-SDU will be sent to the appropriate Service Specific Convergence Sublayer (SSCS) according to the type of higher layer. The IP datagram will be formed which will be segmented (Fig.1-b) to the appropriate frame type (e.g. Frame Relay, Ethernet, ATM etc.), and then will be sent towards the Edge Router.
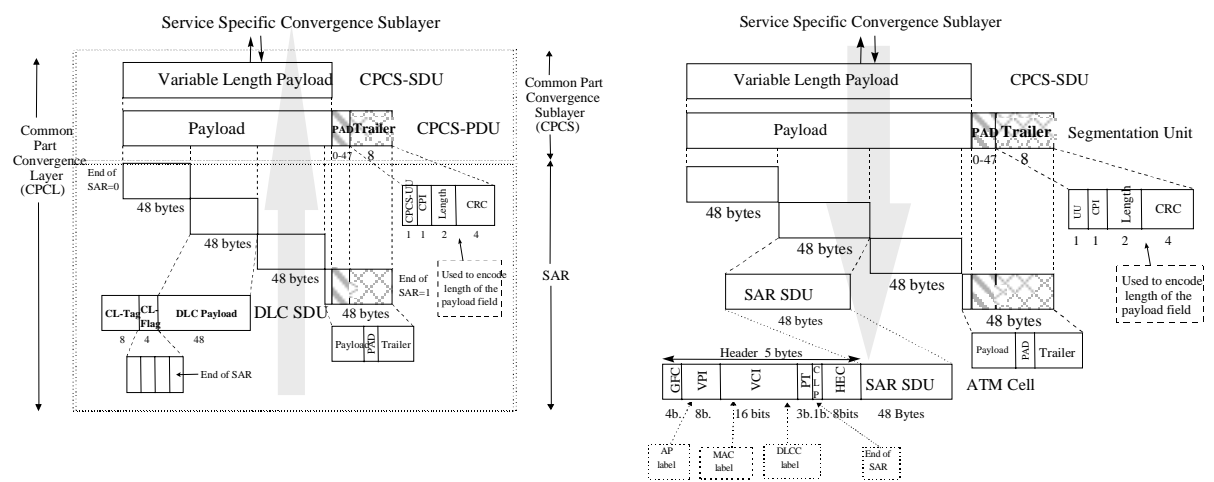

Fig. 1. (a) Reassembly of DLC-PDUs at the AP; (b) Segmentation to form ATM cell, at the AP

However, the VPI/VCI field of the ATM cell will not convey standard ATM information. Since the AP knows that the received DLC-PDU comes from a certain MT, it knows the MAC-ID and the DLCC-ID of the sender. Furthermore, it knows the AP-ID of itself. This information must be mapped into labels, which is inserted in the VPI/VCI fields. The total length of MAC-ID, AP-ID and DLCC-ID is 22 bits, which can be conveyed in the VPI/VCI field (24 bits long). The "End of SAR" information, 
which is retrieved from the last bit of the CL-Flag field, will be inserted in the PT field (see Fig.1-b). The AP knows the MAC-ID of the MT that sends the frames and the ER knows the IP address of this MT. Therefore, they must agree on a label binding to associate this information.

In cases where ATM is used as a layer 2 technology then the re-assembly of DLCSDUs, to form the CPCS-SDU, and re-segmentation to form the ATM cells, is avoided, since the payload in both DLC-PDUs and ATM cells is 48 bytes long. A change in the header, simplifies the entire procedure, i.e. strip the CL-Flag and CLTag fields and insert the ATM header.

The ATM cells can then be transmitted to the ER. The ER receives these ATM cells which are then reassembled to form the IP datagram. The IP datagram is segmented through AAL5 to ATM cells and the ER assigns in the VPI/VCI field the 20-bit label (possibly establishes the LDP path for explicit routing, too). The existing LDP algorithms, as defined by IETF, run at the ER. The ER must have a table to map the received labels from the AP to IP addresses. It must then assign packets to particular Forwarding Equivalent Classes (FEC), map each FEC to a Next Hop Label Forwarding Entry (NHLFE) and finally map each one or a set of NHLFEs to a label. This information will be stored in a Forwarding Information Base (FIB).

The "labelled" ATM cells are now delivered to the core network and according to the labels distributed by LDP, layer 3 switching is performed up to the ER connected to the destined MT.

When a FT or a MT sends a packet to another MT, then the packet will be forwarded according to the IP address. The ingress MPLS router will insert a label according to the destination IP address. The labelled packet will reach the ER which holds all IP addresses of the MTs attached to it. It will assign a label to forward the frame to the AP. Since the AP knows that this label corresponds to the MAC-ID, the frame reaches at its destination.

Downstream. The ER receives labelled ATM cells. The MPLS label is included in the VPI/VCI field and contains information related to the destination and source address (the ER has a table with mappings of MT IP-address to DLCC-label, MAClabel). After passing through AAL5, the cells are reassembled and the IP datagram is constructed. The above information is stored and again through AAL5, ATM cells are formed containing additional information. The VPI/VCI field contains an End of SAR, a DLCC label, a MAC label and a AP label according to the IP addresses, respectively (as illustrated in Fig.1-b).

At the AP, the 5-byte header of the received ATM cells is stripped and the remainder (SAR SDU) is reassembled to form the Segmentation Unit and the CPCSSDU. According to the labels in the VPI/VCI field, the AP has a table, which maps them to MAC-IDs and DLCC-IDs. This way the AP knows the MTs where the DLCPDUs must be forwarded.

The interworking function is responsible for segmenting the CPCS-SDUs to DLCPDUs and mapping the DLCC labels and MAC labels to MAC-IDs and DLCC-IDs. Finally, the DLC-PDUs are transmitted via the air interface to the MT.

The MT receives them and extracts the FU, SN and CRC fields to form the DLCSDUs. From the last bit of the CL-Flag, the end of a message is identified. 


\subsection{AP MPLS Non-capable and ER MPLS Capable}

Upstream. The procedure is the same as described in section 4.1. In this scenario, it is not required to send information to the ER, related to the wireless access part, since the source IP address is known to the ER. Therefore, the ATM cells sent from the AP to the ER contain no labels. The ER receives the ATM cells and through the SAR sublayer, the IP datagram is formed. The ER reads the destination IP address, assigns the packet into a FEC and assigns a label according to that FEC (acts as an ingress router in an MPLS cloud). The MPLS label is encapsulated in the VPI/VCI field of the ATM cell and forwarding is implemented as defined by the IETF's MPLS Working Group.

Downstream. The ER acts as an egress router to an MPLS domain. It reads the label (in the VPI/VCI field of the ATM cells) and maps it to a FEC, which in turn is mapped to an IP address. The ATM cells are reassembled to form the IP datagram. The destination IP address is stored. The ER will have a table to map MT IP addresses to AP IP addresses where the MT is associated with. For this purpose an ATM VP is established from the ER to the AP. The IP datagram is segmented into ATM cells through AAL5 SAR sublayer and will leave the ER destined to the AP.

At the AP, the ATM cells are reassembled, forming the IP datagram and according to the destination address will be forwarded to the appropriate MT, after segmenting the datagram to DLC-PDUs. If there is no connection established with that MT (i.e., AP has not assigned a DLCC-ID to it), a DLCC-ID is assigned and the packet is forwarded to the MT. The MT receives the DLC-PDUs and extracts the FU, SN and CRC fields to form the DLC-SDUs. The last bit of the CL-Flag is used, in order to recognise the last DLC-SDU of the message.

\subsection{Comparison of Two Approaches}

The approach discussed in section 4.1, is advantageous since there is no reassembly of IP datagrams at the AP. This is the reason why the AP maps the MAC-ID to a label and not directly to an IP address. The label will be mapped to an IP address at the ER. Thus, traffic destined for the MT is routed up to the ER according to the IP address (or labels if it is an MPLS Domain connected to the ER), up to the AP according to the labels (of the "light version" LDP) and up to the MT according to the DLCC-ID and the MAC-ID.

Additionally, if the AP is MPLS capable, communication between two MTs attached under the same AP can be realised without involving the ER. This way, the communication between the MTs is faster.

Finally, the second approach has the disadvantage of reassembling the IP datagram at the AP.

\section{Mobility Management in MPLS Domain}

There is a wide range of technologies for supporting mobile users (e.g. GSM, PCS, UMTS, Mobile IP [14]-[16] and Wireless ATM [17],[18]). All these technologies support two fundamental mechanisms: 
- Location management -locating users prior to or during connection establishment

- Handover (HO) -rerouting connections when users move.

Mobility management within IP-based networks could be performed using Mobile IP. However, Mobile IP has certain disadvantages. MPLS could alleviate these as will be described in section 5.1. Moreover, further study has been undertaken to provide Mobility Management using a "Mobility Server" approach within an MPLS Domain (section 5.2). In this case, MPLS signaling protocols (LDP) and MPLS messages are employed to perform mobility management functionalities.

\subsection{Use of Mobile IP}

Current Internet Protocol versions do not support host mobility. IP has been designed such that moving hosts are not considered. Thus, the rise of Mobile IP: an Internet protocol designed to support host mobility [14]-[16]. Mobile IP is consisting of the following entities: Mobile Node (MN), Home Agent (HA), Foreign Agent (FA) and Correspondent Node $(\mathrm{CN})$ and makes use of two addresses, a Care-of-address (COA) and a Home Address.

When a $\mathrm{CN}$ sends a packet to a $\mathrm{MN}$, if the $\mathrm{MN}$ is on its home network the packet will reach the HA and will then be delivered to the MN. If the MN has changed its point of attachment, then the packet will reach the HA. The HA is informed of the new point of attachment of the MN (through the FA), and will forward the packet to the FA that the MN is currently attached. The HA uses an encapsulation process to forward the packet to the FA. It encloses an IP datagram within another IP header, which contains the care-of address of the mobile node. The IP datagram itself remains intact and untouched throughout the enclosing process (Fig.2-a).

Problems with Base Mobile IP Protocol. The problem with this approach is the encapsulation process [19]. When the $\mathrm{MN}$ is located at the foreign network an additional IP header ( 24 bytes) is inserted to the IP packet. This is too much overhead to the final datagram (several fields are duplicated from the inner IP header). This waste of bandwidth is uneconomical. IETF has defined a so-called Minimal Encapsulation scheme, [20], as an alternative to encapsulate the datagram. The approach to the encapsulation method is as follows: Instead of inserting a new header, the original header is modified to reflect the COA. A minimal forwarding header is inserted in between the modified IP header and the unmodified IP payload, to store the original source and destination address. When the FA tries to decapsulate the received IP datagram, it will simply restore the fields in the forwarding header to the IP header, and then remove it. There is a restriction to the use of this encapsulation method. If the original datagram is already fragmented, then minimal encapsulation must not be used since there is no room left to store fragmentation information.

One other problem of Mobile IP regards "triangle routing". Suppose that the CN wants to send a datagram to the MN (Fig.2-b). Based on the base Mobile IP protocol, the $\mathrm{CN}$ will send the datagram to the MN's HA, which may be a half globe away. The HA will then forward the datagram to its COA, which might just take a half second to reach if the datagram is sent directly from the $\mathrm{CN}$. This kind of "indirect routing" is inefficient and undesirable. This problem, can be alleviated through a route optimisation procedure as defined by the Mobile IP Working Group of the IETF [21]. 
The key approach to route optimisation is as follows: Binding cache, containing the mobility binding of MNs, is provided for the $\mathrm{CN}$ that looks for optimising its own communication with MNs. In this way, the $\mathrm{CN}$ has a way to keep track of where the MNs are. So when the $\mathrm{CN}$ wishes to send the datagram to the MN, the IP datagrams are sent directly to the destination address, eliminating the "zig-zag" routing. The means for the MN's previous FA to be notified of the MN's new location is provided. This mechanism allows datagrams in flight to the MN's previous FA to be re-directed to its current address.
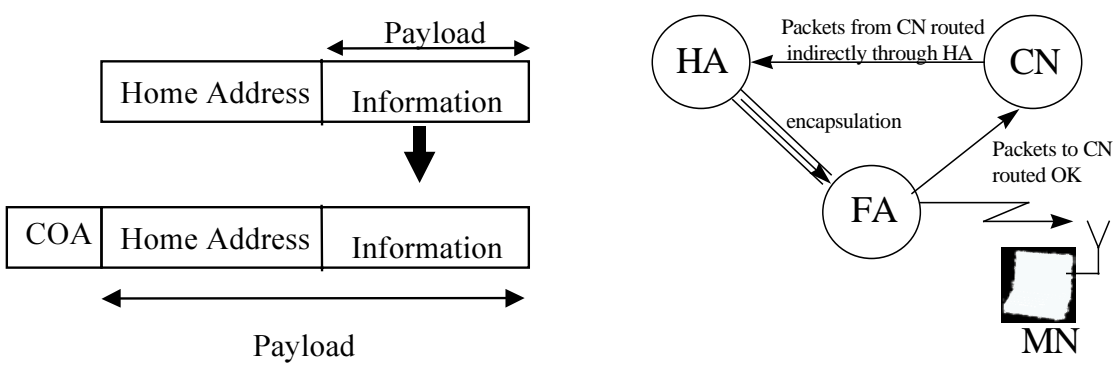

Fig. 2. Problems with base Mobile IP: (a) IP encapsulation; (b) Triangle routing

Solutions Using MPLS. These problems can be alleviated through the employment of MPLS. By making the HA, the FA, the $\mathrm{CN}$ and the MN MPLS capable, we propose the following solutions:

- The "IP-within-IP" encapsulation method can be replaced by the use of label stack offered by MPLS. Forwarding will be based on MPLS labels. Thus, instead of inserting a new IP header ( 24 bytes long), a level 2 label can be used that will be pushed on the label stack by the HA whenever the MN has changed its point of attachment. Therefore, the overhead introduced with MPLS is smaller (MPLS header is 32 bits long) than the overhead introduced by the IP-within-IP encapsulation.

- The "triangle routing" problem can be alleviated according to the following procedure: Suppose that the $\mathrm{CN}$ wants to send a packet to the $\mathrm{MN}$, which is attached to a FA. The CN will send a "label request" message to the HA, using a level 1 label. The HA will forward the "label request" message to the FA by pushing a new level 2 label on the label stack. Since the labelled packet received by the FA arrives only with the level 1 label (the penultimate hop of the FA pops out the level 2 label), the path from the HA to the FA is transparent to the FA. Hence, the FA will send back a "label mapping" message directly to the CN. As a result, an LDP path is established between the $\mathrm{CN}$ and the MN. The $\mathrm{CN}$ sends the packet with the necessary information towards the MN. This way "triangle routing" is only followed by the "label request" message, before the beginning of communication between the $\mathrm{CN}$ and the MN. Furthermore, it is only followed once, prior to the LDP path establishment, and when data is sent, the LDP path is followed, without involving the HA (Fig.3). 


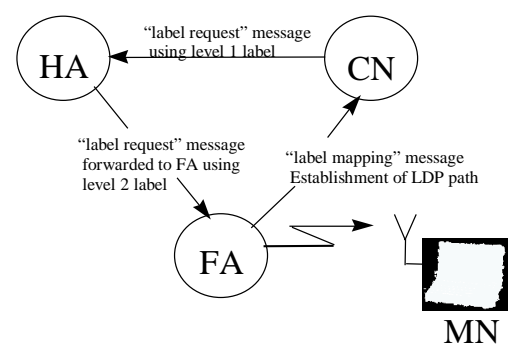

Fig. 3. Alleviation of "triangle routing" using MPLS

\subsection{Using a Mobility Server within an MPLS Domain}

In this approach Mobility Management functions are provided using MPLS signalling protocols (LDP) and MPLS specific messages.

Location Management. Each MPLS Administrative Domain contains a Mobility Server which comprises a Home and a Visitor Database. Both Databases have a table for mapping MT IP addresses to NET-IDs (representing identifier of each MPLS Administrative Domain). The Home Database has entries from MTs belonging to the current MPLS Administrative Domain. The Visitor Database has entries from visiting MTs in the current MPLS Administrative Domain.

Each time a MT powers on, the Mobility Server checks its Home Database. If there is an entry of the MT's IP address it assumes that the MT is located the home MPLS Administrative Domain. Otherwise, the Mobility Server tries to find the MT's "home server", to learn information for the MT and perform authentication. This information for reaching the "home server" will be retrieved by the MT's NET-ID.

All Mobility Servers can be members of a Virtual Private Network (VPN). MPLS provides a simple and efficient solution for VPNs, offering a viable VPN service to the members. This way all Mobility Servers will communicate efficiently, rapidly and with security. Among the Mobility Servers, Constraint Based LSPs will be established. After the Mobility Server has authenticated the MT and received all information from the MT's "home server", it will add an entry in the Visitor Database with the MT's IP address mapped to a NET-ID.

Handover. One of the functional entities of the DLC layer defined by ETSI is Radio link Control Protocol (RCP) [22]. Within RCP, the Radio Resource Control (RRC) entity, is responsible for the HO. There are three different types of HO; Sector HO, Radio HO (intra-AP) and Network HO (inter-AP) which involves the CL and Higher Layers. In the last case, specific signalling is required via the core network. Since the AP is changed, the new and the old APs must communicate to exchange information. Three different types of Network HO have been examined: a) Inter-AP, Inter-ER (Edge Router), b) Inter-AP, Intra-ER and c) Inter-ER, Inter-Domain.

Inter-AP, Inter-ER HO. This case is illustrated in Fig.4-a. When the MT detects the need for a HO to another AP, it may be still synchronised to the current AP. In this case, the MT may notify the current AP that it will perform a HO to another AP. The 
notified AP shall stop transmitting/receiving data to/from this MT, but shall maintain association for a specific time, when indicated.

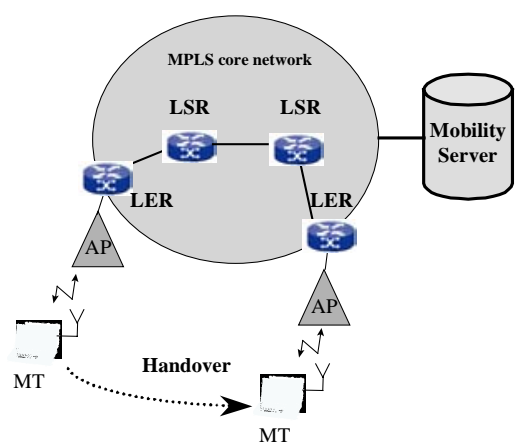

Fig. 4. (a) Inter-AP, Inter-ER Handover

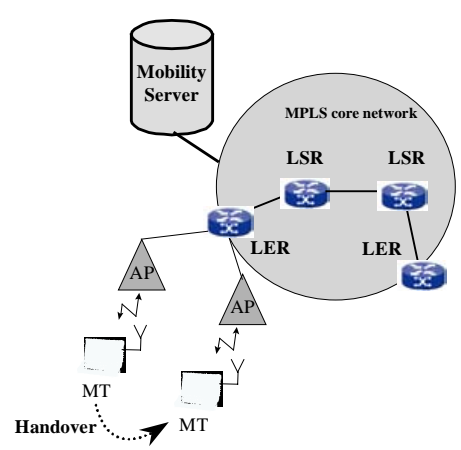

(b) Inter-AP, Intra-ER Handover

Fig.5 illustrates the inter-AP, inter-ER MSC. In the "Handover_Request” message, security parameters such as MAC-ID, NET-ID and AP-ID of the previously connected AP must be indicated. The old AP will be contacted via the backbone network, through the use of MPLS labels. All the parameters related to the on-going connections and security functions must be available in the new AP in order to maintain the association state. These will be sent by the old AP to the old ER and from the old ER to the new one through an LSP tunnel. The new AP negotiates the received parameters and it updates its routing tables about the new point of attachment of the MT.

All traffic sent to the old AP destined for the MT during HO, will be sent to the new AP through the LSP tunnel. This will be kept alive until the explicit LSP path has been established from the new ER towards the CN. The explicit LSP path is established from the new ER by sending a "Label_Request" message to the CN. The CN replies with a "Label_Mapping” message. The explicit LSP path is established and all connections are redirected. The old AP can now perform disassociation of connections with the MT. The LDP_Handover_Alive and LDP_Handover_Reply messages will be sent as LDP specific messages following an explicit or hop-by-hop tunnel. These type of messages are not defined by the MPLS WG, therefore they must be defined. The "Type" range 0x3F00 through 0x3FFF is left by the MPLS WG for extensibility. Following the LDP specification, the encoding scheme used by LDP is Type Length Value (TLV) encoding.

The structure of the "LDP Handover Alive" and "LDP Handover Reply" messages (Fig.6), follow the structure defined by IETF [23]. In addition, it uses Type-LengthValue (TLV) encoding scheme to encode the information carried in LDP messages.

Inter-AP, Intra-ER Handover. For the case where the MT changes AP but the new AP is under the same ER (Fig.4-b), the MSC is illustrated in Fig.7-a.

Inter-ER, Inter-Domain Handover. This scenario is illustrated in Fig.7-b. The new LER tries to find the old one to retrieve relevant information. LERs hold NET-IDs (mapped to IP addresses) of MTs belonging in their MPLS Domain. When the MT has changed Domain it will not find an entry of the NET-ID. Therefore, the LER 
requests from the Mobility Server the NET-ID-IP address binding. The Mobility Server will contact (through the explicit paths between the two members of the VPN) the Mobility Server of the MPLS-Domain where the MT was previously attached. The Mobility Server will then inform the new LER of the IP address of the old LER. Now the new LER can contact the old one as described previously. Therefore, the role of the Mobility Server is to provide the IP address of the LERs that do not belong to the same MPLS-Domain. This way, LERs will have much fewer entries in their tables. They will have entries (NET-ID - IP address binding) only of LERs that belong to the same domain.

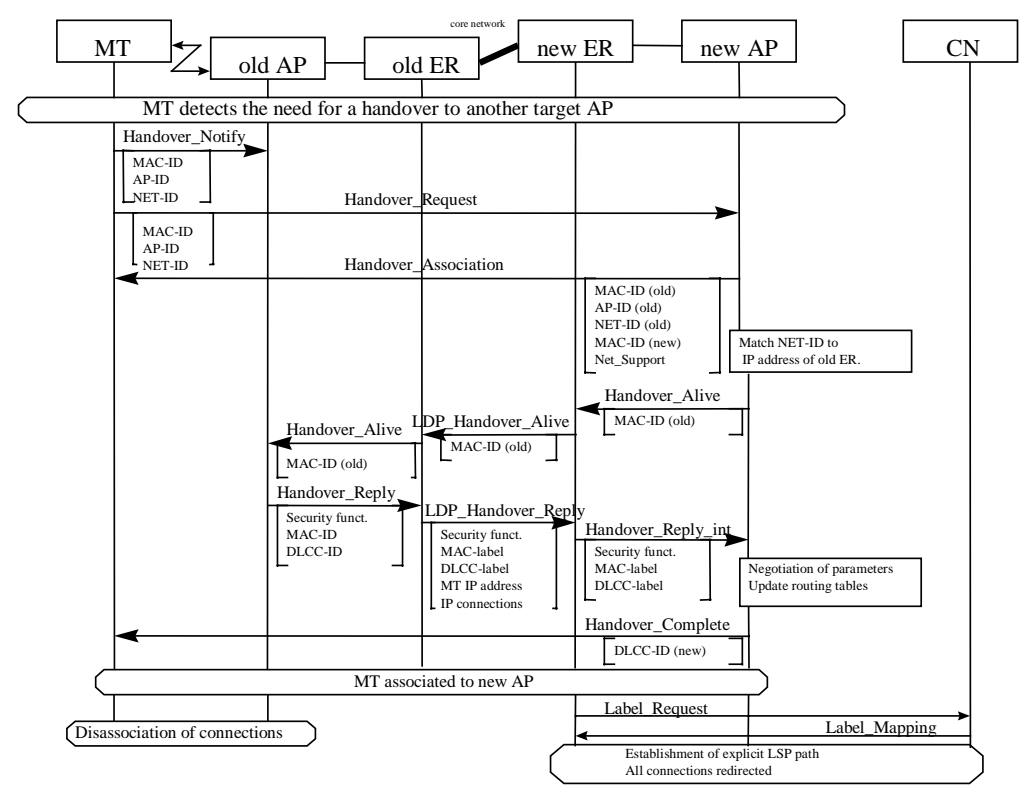

Fig. 5. Proposed Message Sequence Chart for the Inter-AP, Inter-ER case

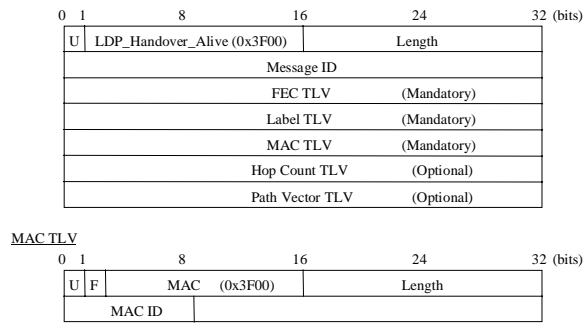

Fig. 6. (a) "LDP Handover Alive"

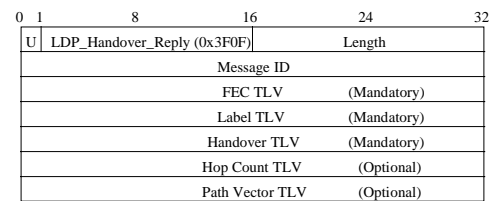

Handover TLV

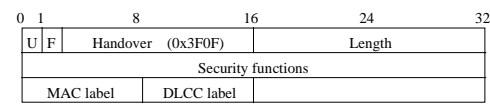

(b) "LDP Handover Reply" 


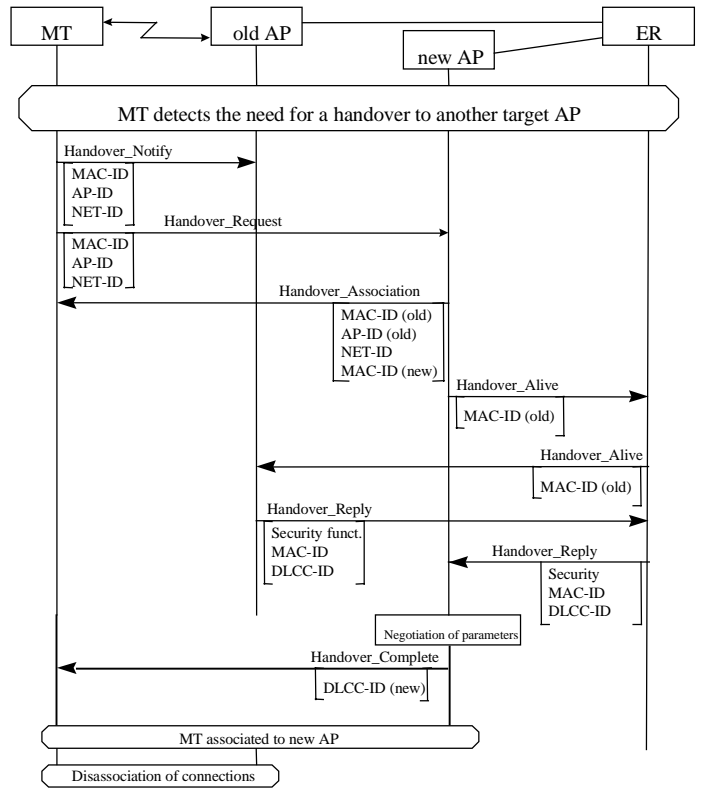

Fig. 7. (a) Proposed Message Sequence Chart for the Inter-AP, Intra-ER case

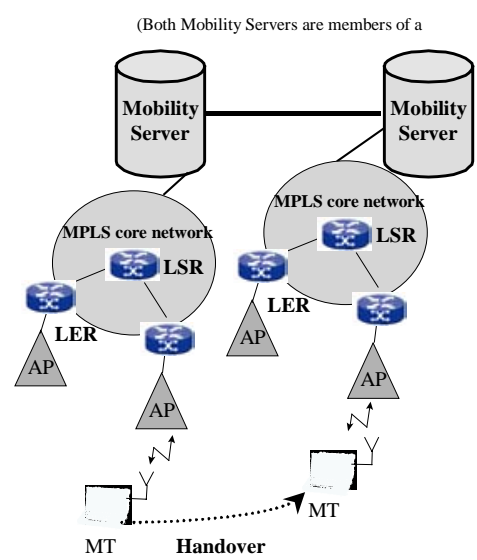

(b)Inter-ER, Inter-Domain Handover

\subsection{Comparison of Two Schemes for Mobility Management}

The first difference between the two approaches is that with Mobile IP there are two addresses: the Home Address and the Care of Address. In the Mobility Server approach there is only one address (like in the HLR/VLR approach of GSM).

Furthermore, in the Mobility Server approach, the "visitor" Mobility Server communicates, via the VPN, with the "home" Mobility Server. After receiving all MT-related information, it updates its database and acts as a "home server". However, with the Mobile IP approach, the HA is informed of the new location of the MT and forwards traffic to the FA which will deliver it to the MT, creating the "triangle routing" problem. Additionally, with route optimisation, the $\mathrm{CN}$ is informed of the new location and not the HA. Hence, it directly sends the packets to the MT, without involving the HA.

\section{Conclusion}

This paper describes how two technologies (MPLS and HL/2) can interwork in order to provide High Speed Wireless Internet access. Two scenarios have been examined. In the first one, the AP is MPLS capable whereas in the second scenario MPLS is terminated at the LER. 
Furthermore, MPLS is employed to provide mobility management. Two schemes have been examined. In the first one, MPLS is combined with Mobile IP to alleviate the problems of IP encapsulation and triangular routing. In the second one, MPLS extensions have been proposed in order to provide mobility management services in an MPLS Administrative Domain through the employment of a Mobility Server. A more detailed analysis of this concept can be found in [24].

\section{References}

1. Ipsilon, "General Switch Management Protocol Spec. v2.0", RFC 2297, March 1998.

2. Rekhter D. et al, "Tag Switching Architecture-Overview", RFC-2105, February 1997.

3. Toshiba Press Release, "Toshiba and Cisco to Cooperate for Next-Generation High Speed Interworking Technology”, http://www.toshiba.co.jp/about/press, 11November 1996.

4. Callon R. et al, "A Framework for MPLS”, Internet Draft, November 1997.

5. Rosen E. et al, "MPLS Architecture", Internet Draft, February 1999.

6. ACTS ITHACI, "IP Switching: Current Technologies and Future Requirements", Deliverable, July 1998.

7. ETSI BRAN, HIPERLAN/2/HIPERLINK, "Requirements and architectures for Wireless Broadband Access and Interconnection", DTR-BRAN-010002v0.1.2, July 1998.

8. ETSI BRAN, "Wireless Access to the Information Age", 1998.

9. ETSI BRAN, HL/2, "System Overview", BRAN_ETR_030002v0.2.0, April 1999.

10. Telia Research, Ericsson, "Outline of the IP Convergence Layer TS", BRAN contribution, HL12.5TRS1a, 15 February 1999.

11. ETSI BRAN, "IP Convergence Layer for HIPERLAN 2 and HIPERACCESS", DTS/BRAN-0024004 v0.0.1, April 1999.

12. Ericsson, "Convergence Layer Basics", BRAN contribution, 3ERI104A, May 1999.

13. ETSI BRAN, "Higher Layer Information IP CL", HL12ERI4A, Ericsson, May 1999.

14. Perkins C. et al, "IP Mobility Support", IETF RFC 2002, October 1996.

15. Perkins C., "Mobile IP: Design Principles and Practices", Addison Wesley, 1998.

16. Becker C. et al, "IP Mobility Architecture Framework", Internet Draft, September 1999.

17. Ayanoglu E. et al, "Wireless ATM: Limits, Challenges and Proposals", IEEE Personal Communications Magazine, August 1996.

18. Acharya A. et al, "Mobility Management in Wireless ATM Networks"

19. Perkins C., "IP Encapsulation within IP", RFC 2003, 6 July 1995.

20. Perkins C., "Minimal Encapsulation within IP", RFC 2004, 6 July 1995.

21. Perkins C., "Route Optimization in Mobile IP", Internet Draft, 25 February 1999.

22. ETSI BRAN, HIPERLAN2; Functional Specification, "Radio Link Control Protocol (RCP) Service Description", Version 2, Revision2, May 1999.

23. Andersson L. et al, "LDP Specification”, Internet Draft, May 1999.

24. Nitsos K., "High Speed Wireless Internet Access: Combination of MPLS and Next Generation Wireless Access Networks (BRAN-HIPERLAN/2)", MSc. Dissertation, September 1999. 